\title{
Itinerario de formación práctica e hito evaluativo: una experiencia para la coherencia, integración y progresión curricular
}

\author{
Itinerary of Practical Training and Evaluation Milestone: An Experience \\ for Curriculum Coherence, Integration and Progression
}

Fancy Castro-Rubilar'; Cristian Rivas-Morales ${ }^{2}$

\begin{abstract}
RESUMEN
Este artículo da a conocer un análisis de contexto y criterios de acreditación para las carreras de pedagogía. Luego, se expone la experiencia de una institución de educación superior en proceso de renovación curricular, dando a conocer algunas estrategias de microdiseño curricular que permiten fortalecer la coherencia, integración y progresión del plan de estudios. El artículo explora, además, los antecedentes teóricos que respaldaron el diseño de dos mecanismos (itinerario de formación práctica e hito evaluativo) y expone una metodología de proceso para el diseño de ambos, para efectos de monitoreo y evaluación del alcance del perfil de egreso y plan de estudios.
\end{abstract}

Palabras claves: acreditación; renovación curricular; integración curricular; perfil de egreso; hito evaluativo; itinerario de formación práctica.

\begin{abstract}
This article presents a context analysis and accreditation criteria for pedagogy careers. Then, the experience of a higher education institution in the process of curricular renewal is exposed, revealing some curricular micro-design strategies that allow to strengthen the coherence, integration and progression of the Curriculum. The article also explores the theoretical background that supported the design of two mechanisms (practical training itinerary and evaluation milestone) and exposes a process methodology for the design of both, for the purposes of monitoring and evaluating the scope of the graduation profile and Curriculum.
\end{abstract}

Keywords: Accreditation; curricular renewal; curricular integration; graduation profile; evaluative milestone; practical training itinerary.

\footnotetext{
${ }^{1}$ Académica del Departamento de Ciencias de la Educación, Universidad del Bío-bío, Chillán, Chile; Doctora en Diseño Curricular y Evaluación Educacional, Universidad de Valladolid, España; fcastro@ubiobio.cl.

${ }^{2}$ Asesor Curricular, Facultad de Educación, Universidad del Bío-Bío, Chillán, Chile; Magíster en Educación, Universidad del Bío-Bío, Chillán, Chile; crivas@ubiobio.cl.
} 


\section{Introducción}

A partir de los años 90, la educación superior, en particular la formación de docentes, ha enfrentado diversos desafíos que en la práctica implican volver a analizar "qué se enseña" y "cómo se enseña". La respuesta a estos desafíos se inicia con proyectos de renovación curricular y actualización de las habilidades de los/as docentes de educación superior con énfasis en fortalecer la "formación de formadores/as". Aun cuando esta tarea está en proceso, las universidades han comenzado en paralelo a desarrollar una segunda renovación, esta vez a la luz de estándares o criterios de calidad. Las culturas institucionales se han visto afectadas por las políticas públicas nacionales, y por las recomendaciones y exigencias internacionales que orientan el quehacer curricular, didáctico y administrativo. En este contexto, encontramos una serie de condiciones que las carreras se concentran en desarrollar, las cuales procederemos a exponer desde las bases teóricas que las sustentan. Posteriormente, presentaremos propuestas de mecanismos de monitoreo del perfil de egreso y de diseño curricular que evidencien integración, coherencia y progresión curricular, y un ejemplo de dichos mecanismos.

\section{Contextos}

Al igual que otras instituciones dependientes del aparato público, las universidades se ven forzadas a demostrar efectividad, la cual siempre está influenciada por fenómenos y factores externos, ya sean económicos, productivos, tecnológicos, sociales o culturales. Con este nuevo objetivo de evidenciar efectividad, los Estados reducen presupuestos y condicionan apoyo financiero a través de fondos concursables, donde se exige el cumplimiento de ciertas condiciones que incluyen definiciones y procesos curriculares.

Las condiciones que exigen los programas de financiamiento público orientan los procesos formativos, cambiando lo que se entiende por objeto de evaluación, los contenidos de la formación y el cómo de la docencia. Entre las condiciones exigidas están, por ejemplo, basar los procesos formativos en modelos por competencia, el uso de ciertas metodologías y tecnologías, y la configuración de planes de estudio de basados en competencias con sus respectivos perfiles de egreso, según profesión. En paralelo, se 
realizan comparaciones internacionales que ponen de relieve lo importante que es la formación superior para la producción económica, social y de capital humano.

Las universidades han debido reorientar su estructura, modelos e interrelaciones para atender las necesidades del mercado y las demandas sociales. La transparencia y la redefinición de "lo público", junto con la democratización de las instituciones, son parte de los itinerarios de las universidades estatales. A finales del siglo pasado, organismos internacionales, diferentes países y, particularmente, la Comisión Internacional sobre la Educación para el Siglo XXI (Unesco, 1996), consideraban de la mayor relevancia transformar los sistemas educacionales, con el objetivo de promover el desarrollo de habilidades en el estudiantado, que se consideraron indispensables para enfrentar los desafíos del nuevo siglo. Se trataba, en consecuencia, de proveer con el capital humano requerido para un desarrollo social sostenible. Estas reformas declaraban la necesidad de promover el aprendizaje a lo largo de la vida y la participación en la sociedad del conocimiento, por medio de la generación y uso colectivo de este último. Sin embargo, los que se consideraban aspectos claves para hacer frente a los desafíos planteados por un mundo en rápida evolución, no llegaron a toda la población de igual manera, manteniendo las brechas educacionales.

El Pacto de Bolonia (1999) estableció como aspectos claves la necesaria articulación entre las universidades y las demandas sociales e industriales que se concretaron en Europa a partir de este hito. Este acontecimiento puso la exigencia a los sistemas universitarios de adaptarse a los desafíos de los postulados de la sociedad del conocimiento y a un mundo globalizado. El hito antecesor de este suceso fue la Declaración de la Sorbona (1998), la cual instaló el concepto de "Espacio Europeo de Educación Superior" (EEES), con el objetivo de lograr en la comunidad la extensión y la calidad de la enseñanza. Para Amtmann (2003), esto refleja la necesidad evidente de un cambio cultural. Por ello, entre las reformas y lineamientos promovidos y acordados en la Declaración de la Sorbona están el establecimiento de tres ciclos educativos (bachillerato, magíster y doctorado), el Sistema Europeo de Transferencia y Acumulación de Créditos, la transparencia en el proceso formativo, cambios en la docencia y evaluación de los procesos de enseñanza y aprendizaje, entre otros. 
En el año 2000, cien universidades de la Unión Europea elaboraron el Proyecto Tuning, apoyado por la Asociación Europea de Universidades (EUA) y financiado en parte por el proyecto Sócrates. Tuning tuvo entre sus principales propósitos los siguientes: otorgar flexibilidad para mejorar los resultados académicos, aumentar la empleabilidad, volver la mirada hacia el/la estudiante como protagonista, permitir diseños diferenciados de programas, propiciar la articulación entre las áreas de conocimiento y entre el pregrado y el posgrado, y promover la movilidad dentro y entre las universidades. Estos objetivos se pusieron en marcha a través de la promoción del diseño de competencias (genéricas y específicas), generando acuerdos respecto a los enfoques de enseñanza, aprendizaje y evaluación, estableciendo créditos académicos transferibles y normando la calidad de los programas.

Cabe aquí hacer una mención breve al origen de la concepción del aprendizaje basado en competencias. Dicha concepción surgió en Inglaterra durante los años 60, en el ámbito laboral y en el contexto de la teoría de la eficiencia social, con el movimiento de educación y entrenamiento con base en competencias (Competence-based Education and Training, CBET) (Hyland, 1994). El modelo propuesto buscaba promover la formación de trabajadores/as para satisfacer las demandas laborales. El concepto fue implementado por David McClelland en los años 70, quien propuso tener más en cuenta las características de los/as trabajadores/as y sus comportamientos concretos ante situaciones de trabajo, en vez de las tradicionales descripciones de atributos, los expedientes académicos y los coeficientes de inteligencia. Detrás del concepto de competencia, la lógica es el aseguramiento de la calidad de la educación superior e implica la capacidad de hacer en contexto (Restrepo et al., 2013). Las definiciones de competencias son muy variadas, tanto como textos publicados al respecto. En este caso lo relevante es que se busca que el/la estudiante sea capaz de evidenciar conocimientos, destrezas y actitudes necesarias para ejercer una actividad laboral, resolver problemas, ser autónomo/a y creativo/a: "Las competencias representan una combinación de atributos (con respecto al conocimiento y sus aplicaciones, aptitudes, destrezas y responsabilidades) que describen el nivel o grado de suficiencia con que una persona es capaz de desempeñarlos" (González y Wagenaar, 2003, p.80). 
El modelo basado o con enfoque en las competencias tiene lógicamente un diseño distintivo. Este pretende asegurar que se centra en los aprendizajes -y, por lo tanto, en los estudiantes y sus contextos-, la transparencia en los procesos y la diversidad de saberes (conocimientos, habilidades, destrezas, valores) necesarios para un desempeño eficiente en contextos específicos. Los modelos curriculares basados en competencias contemplan resultados de aprendizajes centrados en los aprendizajes profundos de los estudiantes (Kri et al., 2015; Pey y Chauriye, 2011). Estos resultados de aprendizaje deben ser operativos, esto es, factibles de ser enseñados, aprendidos y evaluados, lo que evidencia un cambio en el foco de la formación, el cual se traslada desde la enseñanza hacia el aprendizaje.

\section{La renovación de la educación superior en América Latina y Chile}

El Mercosur inició las primeras acciones de internalización de políticas educativas en educación superior en 1998 y, entre los años 2004 y 2007, se ejecutó en América Latina el Alfa Tuning América Latina (Tunning AL), en total consonancia con el proyecto Tuning de Europa. Uno de los trabajos principales implicó la revisión y análisis de perfiles de egreso coherentes, centrándose en las estructuras y el contenido de los estudios ${ }^{3}$. En América Latina las universidades involucradas trabajaron en consorcio, bajo financiamiento estatal y auspicio internacional, específicamente del proyecto ALFA. En total, participaron 62 universidades latinoamericanas que trabajaron en cuatro áreas: Administración de Empresas, Educación, Historia y Matemáticas. En este proceso, las universidades, a pesar de las orientaciones internacionales de consenso que van configurando su actuar, conservan su diversidad y autonomía (Bravo, 2007). Tuning AL confirma la relación fundamental de la universidad con el desarrollo social y económico, $\mathrm{y}$, por consiguiente, la necesidad de ajustar, permanentemente y bajo retroalimentación

\footnotetext{
${ }^{3}$ Este trabajo fue desarrollado por ocho universidades latinoamericanas: Universidad Nacional de La Plata (Argentina), Universidad Estadual de Campinas (Brasil), Universidad de Chile, Universidad de Costa Rica, Pontificia Universidad Javeriana (Colombia), Universidad Católica Andrés Bello (Venezuela), Universidad Rafael Landívar (Guatemala) y Universidad de Guanajuato (México). Junto a ellas, trabajaron siete universidades europeas: Technische Universität Braun-scheweing (Alemania), Universidad de Deusto (España), Universidad Paris IX-Dauphine (Francia), Universidad de Pisa (Italia), Universidad de Groningen (Países Bajos), Universidad de Coimbra (Portugal) y Universidad de Bristol (Reino Unido).
} 
externa, los planes de estudio y perfiles según las necesidades del contexto local y global (González y Wagenaar, 2004).

En la primera reunión de Alfa Tuning AL, realizada en marzo de 2005 en Buenos Aires, las universidades participantes lograron definir la lista de competencias específicas para las áreas temáticas de Administración de Empresas, Educación, Historia y Matemáticas. Posteriormente, siguieron la misma ruta que Europa, con principios y conceptualizaciones comunes, tales como competencias según área de desarrollo, enfoques específicos de enseñanza, aprendizaje y evaluación, sistema de créditos transferibles (SCT), y criterios de calidad de los programas y carreras. Otro cambio fundamental que se se realizó consistió en pasar desde el foco en los contenidos y objetivos de el/la profesor/a, hacia una orientación centrada en los/as estudiantes, lo que exige una estructura de programas basada en resultados de aprendizaje, uso específico de taxonomías y estrategias metodológicas y de evaluación, afectando los diseños macro y microcurriculares (Bravo, 2007)

A fines de la década de los 90, Chile inicia reformas de la educación superior, buscando mayor acceso y calidad. Asimismo, fiel a su estilo de desarrollo, incorpora elementos de Bolonia, Tuning y la experiencia norteamericana de educación superior, incluyendo el modelo de competencias generales y específicas de Quebec (Canadá) y el sistema europeo de créditos transferibles y acumulables (ECTS). Las reformas fueron financiadas por el Banco Mundial, bajo los proyectos Mecesup (Mejoramiento de la Equidad y Calidad de la Educación Superior) del Ministerio de Educación. En el contexto de la ejecución de estas reformas, se esgrimen, entre otros, argumentos de índole productiva, de efectividad y mejora de rendimientos, así como la necesidad de crear competencias para el desarrollo social, cultural y medioambiental.

Curricularmente, las renovaciones implican un cambio de los procesos de enseñanzaaprendizaje, pasando desde los contenidos a los resultados de aprendizaje y desde una estructura fragmentada a una integrada. La cultura institucional gremial y fragmentada, propia de modelos industriales (Yániz y Villardón, 2006), se verá confrontada por estas nuevas formas complejas, colaborativas y contextualizadas 
El proceso de renovación curricular implicó un cambio de mentalidad, modificando, entre otros, el enfoque pedagógico: pasar del enseñar al aprender, considerar las demandas del medio social, natural y cultural, así como el trabajo interdisciplinario y colaborativo (Solar-Rodríguez, 2005).

En particular, para el caso de la educación superior, Solar-Rodríguez (2005) señala que

las competencias pueden ser una estrategia especialmente indicada para los aspectos fundamentales del diseño: se identifica el diseño de la parte profesional de los planes de estudios y el diseño general en lo que atañe al desarrollo de competencias genéricas, como el trabajo en equipo, comunicación, responsabilidad social y otras. (p.182)

En tanto, para Tobón (2006), lo que caracteriza al enfoque por competencias es que en vez de poner el acento en conocimientos conceptuales y factuales, privilegia el desempeño integral ante actividades y problemas.

Las competencias entregan, en definitiva, un marco conceptual común para la definición de perfiles y modelos de aprendizaje. Lo anterior tiene implicancias epistemológicas y puede modelar los procesos educativos, entregando un marco de calidad para el diseño curricular y para la definición de los desempeños esperados.

\section{Calidad en la formación docente como eje de procesos de renovación curricular}

Los modelos basados en competencias permean las políticas de calidad, lo cual se observa en los criterios de acreditación de las carreras de pregrado (Comisión Nacional de Acreditación y Ministerio de Educación, 2008). Se busca asegurar la consistencia interna y externa de los programas, siendo la acreditación una herramienta de gestión que debería optimizar y mejorar los procesos de un diseño curricular consistente, con coherencia entre perfil, plan de estudio y programas, así como sus estrategias y evaluación. 
La formación de formadores/as cobra relevancia, ya que el mayor impacto sobre el aprendizaje de los/as estudiantes radica en las prácticas docentes y su efectividad al interior de la sala de clases (Pedraja et al., 2012). El año 2008 comenzaron a emerger en Chile las políticas de calidad para la formación inicial docente, con la elaboración de los estándares pedagógicos para los/as egresados/as de las carreras pedagógicas en educación básica y, en el 2010, para las carreras de educación media. Se entiende estándar como lo que todo/a docente debe saber y poder hacer para ser considerado/a competente. Los estándares ministeriales constituyen insumos para orientar la formación pedagógica en las instituciones formadoras y son claves para el diseño de perfiles de egreso, requisitos y mallas curriculares (Ministerio de Educación [Mineduc], 2012). Estos estándares se encuentran definidos en la nueva Ley General de Educación (2009) y comunican a la sociedad, en especial al campo de las carreras profesionales y académicos/as, una visión de cuáles son las competencias que los/as profesionales de la docencia deben poseer en las distintas disciplinas (Lenguaje y Comunicación; Matemáticas; Historia, Geografía y Ciencias Sociales; Biología; Física; Química).

Los estándares contemplan un conjunto de indicadores que describen disposiciones y valoraciones, desglosando y especificando de qué modo se manifiesta el logro de conocimientos y habilidades correspondientes al desempeño esperado de los/as futuros docentes. A las orientaciones disciplinares se suman orientaciones de dominios y competencias "pedagógicas", que corresponden a conocimiento del currículo, diseño de procesos de aprendizaje y evaluación, así como de ciencias sociales vinculadas como psicología, sociología y filosofía. También se incluyen elementos para la gestión y administración de la cultura escolar, tales como el Marco para la Buena Enseñanza y el Marco para la Buena Dirección y el Liderazgo Escolar.

Entre los años 2010 y 2014, el Programa Inicia buscó fomentar la calidad de la formación inicial docente con tres estrategias: a) definición de estándares e instrumentos técnicos que describen lo que cada docente debe saber de la disciplina y su enseñanza; b) implementación de una evaluación de conocimientos y habilidades para egresados/as de las carreras de pedagogía, que entrega información a las instituciones y a los/as evaluados/as sobre el logro de los estándares; c) recursos concursables para ejecutar proyectos de mejoramiento de las carreras de pedagogía (Mineduc, 2012). 
Por su parte, el Marco Nacional de Cualificaciones (Mineduc, 2016) otorga un marco para estandarizar y homologar condiciones mínimas de desempeño a nivel nacional, tanto en pre como posgrado. Cabe destacar que los descriptores se relacionan directamente con los resultados de aprendizaje y estos provienen de competencias declaradas. El Marco Nacional de Cualificaciones otorga definiciones acorde a los títulos y grados de educación superior, contribuyendo al diseño de los perfiles de egreso, expresando en competencias $\mathrm{y}$ habilidades el nivel de desempeño pertinente (licenciatura y profesional de nivel avanzado, que es el nivel tres). El Marco Nacional de Cualificaciones no solo define el nivel de conocimientos, habilidades y competencias, también establece el volumen de aprendizaje (tiempo en SCT-Chile), que se sitúa entre los 240 SCT y 300 SCT para la categoría de profesional avanzado.

En general, en los estándares orientadores para carreras de pedagogía (CPEIP, 2019), se espera que el/la egresado/a brinde soluciones a problemas concretos y que comprenda el contexto más allá de niveles puramente técnicos. En cuanto a conocimientos, se espera que sea capaz de demostrar el conocimiento disciplinar. Respecto a las habilidades, se espera que pueda reflexionar e integrar información y resolver problemas, diseñar propuestas, comunicar de manera efectiva y colaborar en tareas de investigación bajo principios de trabajo colaborativo, ética, autonomía y respeto por la diversidad.

Como hemos señalado, el financiamiento de proyectos muchas veces actúa como un incentivo para que las universidades incorporen las políticas y orientaciones emanadas del Estado. Entre esos incentivos estuvieron los proyectos Mecesup (1999-2015), con convenios de desempeños (2007-2015) en los que las universidades diseñaron nuevos perfiles y planes de estudio, bajo el nombre de renovaciones curriculares (Cisterna et al., 2016). Las renovaciones curriculares no solo promovían los modelos basados en competencias a principios de la década del 2000, sino que reforzaban el uso de nuevas prácticas pedagógicas, las cuales en muchos casos eran desconocidas y resistidas por los/as académicos/as, quienes en su mayoría expresaban no estar preparado para un modelo por competencias (Espinoza, 2014).

Con todo, los/as académicos/as y docentes siguen viendo la renovación curricular como un escenario complejo, que va desde la definición de competencias hasta el bosquejo de elementos de evaluación, para comprobar si los/as estudiantes lograron las 
competencias esperadas. La renovación curricular exige también compromiso y liderazgo para asumir los desafíos en un marco de innovación. La definición de perfiles, los diseños de las estrategias evaluativas y la construcción de programas de asignatura son procesos complejos, sobre los cuales además hay criterios y principios establecidos por la política pública, entre los que se exige que los diseños curriculares sean validados por partes interesadas como académicos/as pares, agentes de organismos públicos, empleadores/as, egresados/as, entre otros actores, bajo criterios de coherencia y pertinencia respecto al contexto y las necesidades (López, 2011).

Conceptos como coherencia, integración, progresión, consistencia, desempeños, se suman ahora como elementos emergentes y fundamentales para un diseño efectivo de renovación o ajuste curricular. Esto en un marco donde la calidad pasa a ser una política institucional y donde se despliegan esfuerzos sistémicos de las instituciones, enfrentando el desafío de lograr el cambio cultural requerido para avanzar hacia una educación superior y formación docente que sean efectivas. La coherencia no es solo interna o microcurricular en los programas de asignatura, sino que también se aplica a los itinerarios o trayectorias formativas, la gobernanza y liderazgo en las instituciones, y la relación entre los desempeños y lo que se espera en los establecimientos escolares, con nuevos desafíos de interculturalidad y multiculturalidad, género, inclusión y protección del medio ambiente.

Los sistemas y modelos de aseguramiento de la calidad corresponden a un conjunto de mecanismos y procesos que buscan garantizar y promover la calidad en las instituciones de educación superior, permitiendo también posicionar a dichas instituciones y generar confianza en la comunidad. El Sistema de Aseguramiento de la Calidad de la Educación Superior (Sinaces) se configuró con la Ley 20.129, la cual contiene dimensiones, criterios y estándares, con un total de 92 descriptores, que consideran docencia y resultados del proceso de formación, gestión estratégica y recursos institucionales, aseguramiento interno de la calidad orientado al mejoramiento continuo (resguardando el desarrollo integral y armónico del proyecto institucional), vinculación con el medio, investigación, creación y/o innovación.

En Chile, con la Resolución Exenta Nº DJ 009-4, promulgada el 3 de agosto 2015, se aprueban los criterios de evaluación para la acreditación de carreras profesionales, 
profesionales con licenciatura y programas de licenciatura. Específicamente, se trata de tres dimensiones y doce criterios, algunos de los cuales tienen especificaciones para el caso de las carreras de pedagogía, como los criterios presentados en la Agrupación de Universidades Regionales (AUR) 2017. Los criterios y estándares para las carreras de pedagogía avanzan en 2019, al establecer estándares óptimos por cada descriptor y evidencias pertinentes.

En 2018, los resultados de los procesos de acreditación para las carreras de pedagogía evidenciaron que el 64\% estaba acreditada, contando con un total de 77.721 estudiantes. Entre las regiones de Bío Bío y Nuble existían 114 programas, de los cuales el 55\% estaba acreditado, contando con 12.726 estudiantes en total, de los cuales el $78 \%$ pertenecía a instituciones acreditadas. En cuanto a los resultados de cumplimiento de estándares, los procesos de acreditación entre el 2016 y 2017, tras la Ley 20.903 sobre desarrollo profesional docente, evidenciaron 2.013 referencias de fortalezas, de las cuales el $80 \%$ se concentró en las dimensiones "perfil de egreso y resultados" y "condiciones de operación", mientras que de las 1.019 debilidades encontradas, el 50\% también se concentró en estas mismas dimensiones.

Existen criterios de evaluación que aparecen como críticos dentro de los procesos de acreditación para los programas de pedagogía. Las unidades académicas deben estructurar su currículum en función de los estándares descritos en los criterios de calidad; se debe evidenciar que el plan de estudios y los programas son consistentes con los principios, objetivos, perfil de egreso y competencias mínimas declarados por la carrera, que deben ser conocidos por los/as estudiantes. Respecto a los resultados de los procesos de acreditación realizados entre 2016 y 2017, dentro de las debilidades detectadas en materia de estructura curricular, destacó la investigación educativa, el plan de estudios y competencias, la formación disciplinar y pedagógica, y la formación práctica. En cuanto al criterio de autoevaluación, las debilidades relativas a la calidad y la capacidad de autorregulación fueron las que aparecieron con mayor frecuencia.

Las debilidades halladas en los procesos de acreditación de los programas de pedagogía son coherentes con otros procesos de revisión de cumplimiento de estándares, como son los resultados de la Evaluación Nacional Diagnóstica II, la cual es rendida anualmente por más de 4.000 estudiantes, en los dos semestres que anteceden al último 
año. Dicha evaluación está compuesta por secciones de selección múltiple y de respuesta abierta, las que miden conocimientos pedagógicos generales para cada nivel educativo, así como conocimientos disciplinares y didácticos. Adicionalmente contiene un cuestionario de información complementaria (Centro de Perfeccionamiento, Experimentación e Investigaciones Pedagógicas [CPEIP], 2012).

El ítem de conocimiento pedagógico de la Evaluación Nacional Diagnóstica II realizada en 2019, en general, mostró descensos en comparación con los años 2017 y 2016, especialmente en el tema "Profesión docente y el sistema educacional chileno" (CPEIP, 2019). En cuanto al logro de estándares, en general, los más bajos corresponden a la conducta profesional, a la aplicación de métodos de evaluación para observar el progreso de los/as estudiantes y a la utilización de los resultados para retroalimentar el aprendizaje y la práctica pedagógica.

Con ello podemos observar que, en general, la toma de decisiones didácticas y evaluativas, así como el ítem de profesión docente que forma parte de todos los perfiles de egreso, es donde más debilidades existen. Las razones están en los mismos criterios que se exigen en los procesos de acreditación, que son la coherencia, la integración y progresión de los planes de estudio.

Al año 2018, el 22 \% de los/as chilenos/as ingresó a la educación superior, 15 puntos más abajo que la media de la OCDE (CNED, 2019). Para el 2019, la matrícula total en educación superior fue de 1.180 .181 personas. Un 93\% de esta matrícula se concentró en universidades acreditadas.

La formación inicial docente se configuró desde el año 2016 con la Ley 20.903, la cual crea el Sistema de Desarrollo Profesional Docente. Esta iniciativa es parte de un conjunto de políticas y estrategias a nivel nacional que, desde los años 90, buscan restituir el estatus de la profesión docente y mejorar las remuneraciones y el desarrollo profesional (Comisión Nacional de Acreditación [CNA], 2015). La ley establece un plan de desarrollo a diez años (2016-2026) que busca mejorar la calidad de la formación inicial docente, así como desarrollar una carrera docente que mejore el valor social de la profesión. Fundamental es que esta ley establece que la acreditación de las pedagogías debe ser obligatoria, debiendo obtenerse como mínimo dos años de acreditación. El proceso de 
acreditación de las carreras de pedagogía asume el desafío de cumplir con estándares nacionales e internacionales para la formación docente, respecto a los propósitos institucionales, el diseño curricular, las condiciones de operación, los resultados del proceso formativo y la capacidad de autorregulación

En definitiva, las carreras de pedagogía deben enfrentar dos procesos de diseño altamente complejos, como son las renovaciones curriculares y la implementación de procesos de calidad, que significan una mejora y ajuste permanente. Para realizar estos constantes ajustes es necesario contar con principios definidos, consensuados y propios de la cultura institucional, así como con criterios de alineamiento curricular, los cuales han de facilitar la integración de áreas del saber en un plan de estudio, permitir la progresión de desempeños y otorgar coherencia al proyecto de renovación, asegurando una sólida formación profesional.

La integración curricular supone una proyección cualitativamente diferente e impone al diseño curricular el reto de integrar los conocimientos, las habilidades y la competencia en el ejercicio de la profesión, estimulando la creatividad y la capacidad de decisión para la solución de situaciones complejas y novedosas. (Vecino, 1999, citado en Ferreira 2010, p.5)

En esta línea, resulta fundamental contar con estrategias de alineamiento curricular (Liu, 2009) que permitan lograr una visión integrada de lo que debe ser enseñado y evaluado. El alineamiento curricular es un principio central y altamente estudiando en su efectividad. Para que este alineamiento sea posible, es necesario que exista una relación lógica entre lo declarado en cuanto a contenidos y habilidades, así como coherencia entre los estándares y mecanismos de evaluación (Bhola et al., 2005; Panuel, 2012). Además, debe existir integración curricular, coherencia y progresión dentro del plan de estudios. Esto es, una relación lógica entre las asignaturas, repetición de estrategias metodológicas y evaluativas, y un desarrollo progresivo de habilidades para resolver problemas en distintos contextos profesionales (Hinde, 2005; Muller, 2009; Beane, 1997).

A continuación, presentaremos algunas estrategias curriculares diseñadas en el marco de los procesos de renovación curricular de la Universidad del Bío Bío y la 
implementación de procesos de aseguramiento de calidad que den cumplimiento a los procesos de acreditación.

\section{Integración y alineación curricular en itinerarios formativos y programas de asignatura}

Como hemos mencionado con anterioridad, los desafíos a los cuales se ven obligadas las instituciones de educación superior y sus criterios de acreditación se confrontan con las prácticas, inercias y rutinas propias de las esferas académicas. No obstante, consideramos pertinente y efectivo desarrollar en el diseño curricular elementos de integración.

Para el diseño de programas e itinerarios alineados y, por lo tanto, integrados, coherentes y progresivos, que sean capaces de fortalecer las líneas de didáctica y profesión docente en el marco del cumplimiento de perfiles de egreso, es necesario adoptar estrategias curriculares desde el diseño de los programas de asignatura, en virtud del logro del perfil de egreso. Cabe destacar que el perfil de egreso es "un conjunto de conocimientos, competencias y actitudes que el estudiante de la carrera o programa habrá internalizado al momento de su titulación o graduación, y constituye el marco de referencia para la aplicación de los créditos de evaluación" (CNA, 2015, p.4).

El primer elemento de desarrollo es a nivel microcurricular con los resultados de aprendizaje. Al desarrollar esta intervención bajo una lógica de alineación constructiva (Biggs, 1996), aseguramos procesos que integran las distintas áreas del saber y la evaluación de desempeños progresivos. Así, en el diseño de los programas de asignatura de especialidad, que superan el $50 \%$ de las asignaturas de un plan de estudios, se orientan resultados de aprendizaje de carácter pedagógico, incluyendo en los contenidos y criterios de evaluación elementos actitudinales, propios de las competencias genéricas.

En la asignatura Pueblos Originarios de América y Chile, por ejemplo, se diseñaron los siguientes resultados de aprendizaje: a) Comprende la presencia del hombre en América y Chile, con la finalidad de observar las diversas formas de adaptación al medio; b) Identifica los aportes culturales a la humanidad de los diversos pueblos y culturas que existieron en América y Chile; c) Reconoce los principales conflictos que viven hoy los 
pueblos originarios de América y Chile; d) Reconoce en los pueblos originarios los legados culturales que aún perviven en medio de la sociedad contemporánea y se compromete con la difusión y defensa de los mismos; e) Elabora material didáctico que posibilite una mayor compresión de los contenidos de la asignatura.

Este ejercicio se debe desarrollar en diversas asignaturas de especialidad, las cuales, en conjunto con asignaturas del área pedagógica (que especifican competencias pedagógicas que conforman la licenciatura) y asignaturas del área genérica (competencias del sello institucional), se alinean con la trayectoria de asignaturas prácticas, como se verá en la Tabla 1.

\section{Tabla 1}

Asignaturas que incorporan resultados de aprendizaje de carácter didáctico y pedagógico

\begin{tabular}{|c|c|c|c|c|c|}
\hline $\mathbf{N}^{\circ}$ & Asignatura & 纪 & 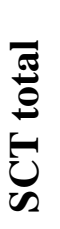 & 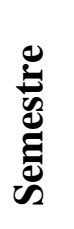 & Resultado de aprendizaje (RA) que tributa \\
\hline 1 & $\begin{array}{l}\text { Pueblos } \\
\text { Originarios } \\
\text { de América } \\
\text { y Chile }\end{array}$ & 2 & 5 & II & $\begin{array}{l}\text { RA5. Elabora material didáctico que posibilite } \\
\text { una mayor compresión de los contenidos de la } \\
\text { asignatura. }\end{array}$ \\
\hline 2 & $\begin{array}{l}\text { Economía y } \\
\text { Desarrollo } \\
\text { Sustentable }\end{array}$ & 2 & 6 & III & $\begin{array}{l}\text { RA 4. Diseña estrategia didáctica para } \\
\text { incentivar la creación de soluciones a los } \\
\text { problemas mundiales, a la conformación de } \\
\text { espacios económicos y modelos de desarrollo, } \\
\text { utilizando métodos de interpretación de datos } \\
\text { y representación espacial que propongan un } \\
\text { desarrollo sustentable desde la economía. }\end{array}$ \\
\hline 3 & $\begin{array}{l}\text { Geosistemas } \\
\text { Naturales II }\end{array}$ & 2 & 4 & III & $\begin{array}{l}\text { RA 5. Utiliza técnicas y métodos de análisis } \\
\text { geográfico orientado hacia una enseñanza } \\
\text { holística del geosistema terrestre, que facilite } \\
\text { el proceso enseñanza-aprendizaje de los/as } \\
\text { estudiantes. }\end{array}$ \\
\hline
\end{tabular}


4 Historia

Medieval

5 Geografía

Regional

6 Historia

Moderna

Chile del

Siglo XIX
7 Historia de

24

III

4. Reconoce diversas estrategia metodológicas para la enseñanza de la historia medieval.

25

IV

RA 4. Elabora propuesta didáctica para enseñar los contenidos del curso relativos a la regionalización mundial y los procesos de globalización.

$24 \quad$ IV

RA 6. Aplica diversas herramientas de enseñanza con el fin de crear conciencia y compromiso con el legado del período moderno para la comprensión de la cultura occidental.

$\begin{array}{lll}2 & 5 & \mathrm{~V}\end{array}$

RA 3. Formula síntesis y análisis de este período de nuestra historia patria, con los propósitos de una mejor compresión de los acontecimientos y una adecuada formación profesional.

8 Historia de $24 \quad 4 \quad$ V América del siglo XIX

9 Geografía Regional de América
10 Historia Mundial Contemporánea del Siglo XIX

\section{Memoria} Histórica y Ciudadanía

12 Historia de 2 Chile Actual
RA 3. Reconoce diversas estrategias metodológicas para la enseñanza de la historia de América del siglo XIX.

$25 \mathrm{~V}$

RA 5. Elabora propuesta didáctica para enseñar los contenidos del curso: sistema natural de América, interrelación sociedad, medio, evolución y desarrollo del territorio impacto medioambiental, etc., en contextos educativos primarios o secundarios, según las bases curriculares establecidas por el Ministerio de Educación.

$24 \quad \mathrm{~V}$

RA 3. Desarrolla el pensamiento histórico, a través del trabajo con fuentes primarias y secundarias.

RA 3. Investiga un tema controvertido de la historia chilena reciente y diseña propuesta didáctica factible de aplicar en contextos escolares de educación media.

5 VI RA 3. Reconoce diversas estrategias metodológicas para la enseñanza de la historia de Chile de los siglos XX y XXI. 
13 Historia de $2 \quad 5 \quad$ VI RA 3. Aplica diversas herramientas de América Contemporánea

14 Sistema Natural Chileno

RA 4. Aplica diversas herramientas de enseñanza con el fin de crear conciencia y compromiso con la vida cívica del Chile actual. enseñanza con el fin de difundir la diversidad de problemáticas que comprende la historia contemporánea de América Latina.

\section{VI $\quad$ RA 4. Elabora propuesta didáctica para} enseñar los contenidos del curso: unidades naturales, riesgos físicos y sistema territorial, en contextos educativos primarios o secundarios, según las bases curriculares establecidas por el Ministerio de Educación.

15 Historia

24

RA 3. Desarrolla el pensamiento histórico, a Mundial Contempotravés del trabajo con fuentes primarias y secundarias.

ránea

16 Problemáti-

24

VII cas sociales de América

y Chile Contemporáneo

17 Sistema

Humano

Chileno

18 Historia

Regional

RA 3 Aplica diversas herramientas de enseñanza con el fin de crear conciencia y compromiso con la vida cívica del Chile actual, en perspectiva latinoamericana.

RA 3. Elabora propuesta didáctica para enseñar los contenidos del curso (división administrativa, actividades económicas, sociodemográfica, impacto medioambiental y sistema humano), en contextos educativos primarios o secundarios, según las bases curriculares establecidas por el Ministerio de Educación.

19 Ciudadanía $2 \quad 4 \quad$ VIII y su Enseñanza

RA 3. Utiliza la tecnología educativa para diseñar recursos de aprendizaje que releven el valor y la importancia de los tesoros humanos vivos de la región.

RA 3. Formula un plan de mejoramiento de la convivencia democrática en contextos escolares, basado en principios como bien común, solidaridad, equidad, igualdad, respeto y justicia, entre otros. 
RA 4. Investiga una problemática asociada a la relación existente entre ejercicio ciudadano y espacio público, con la idea de entregar propuestas que consideren estrategias y metodologías de mejoramiento de los espacios donde es posible desarrollar una ciudadanía plena.

\section{Total} $36 \quad 83$

Nota: esta contribución desde las asignaturas de especialidad muestra la integración de la disciplina con elementos pedagógicos que confluyen en las asignaturas de práctica, configurando lo que se ha denominado "itinerario de formación práctica".

\section{Tabla 2}

Itinerario de formación práctica. Asignaturas que fortalecen el área didáctica desde la disciplina

\begin{tabular}{|c|c|c|c|c|c|c|c|}
\hline $\begin{array}{l}\text { I } \\
\text { Semes- } \\
\text { tre }\end{array}$ & $\begin{array}{l}\text { Introduc- } \\
\text { ción a la } \\
\text { Geogra- } \\
\text { fía }\end{array}$ & $\begin{array}{l}\text { Historio- } \\
\text { grafía }\end{array}$ & $\begin{array}{l}\text { Técnicas } \\
\text { en Geo- } \\
\text { grafía }\end{array}$ & $\begin{array}{l}\text { Primeras } \\
\text { Civiliza- } \\
\text { ciones }\end{array}$ & $\begin{array}{l}\text { Filosofía } \\
\text { Educa- } \\
\text { cional }\end{array}$ & $\begin{array}{l}\text { Sociología } \\
\text { de la } \\
\text { Educación } \\
\text { con } \\
\text { Enfoque } \\
\text { de Género }\end{array}$ & $\begin{array}{l}\text { Compe- } \\
\text { tencias } \\
\text { Comuni- } \\
\text { cativas } \\
\text { FIP }\end{array}$ \\
\hline $\begin{array}{l}\text { II } \\
\text { Semes- } \\
\text { tre }\end{array}$ & $\begin{array}{l}\text { Geo- } \\
\text { grafía } \\
\text { Humana }\end{array}$ & $\begin{array}{l}\text { Pueblos } \\
\text { origina- } \\
\text { rios de } \\
\text { América y } \\
\text { Chile }\end{array}$ & $\begin{array}{l}\text { Geosis- } \\
\text { temas } \\
\text { Natura- } \\
\text { les I }\end{array}$ & $\begin{array}{l}\text { Historia } \\
\text { Clásica }\end{array}$ & $\begin{array}{l}\text { Tecnolo- } \\
\text { gías para } \\
\text { el Apren- } \\
\text { dizaje }\end{array}$ & $\begin{array}{l}\text { Sociedad } \\
\text { Cultura y } \\
\text { Educa- } \\
\text { ción }\end{array}$ & $\begin{array}{l}\text { Psicolo- } \\
\text { gía Edu- } \\
\text { cacional }\end{array}$ \\
\hline $\begin{array}{l}\text { III } \\
\text { Semes- } \\
\text { tre }\end{array}$ & $\begin{array}{l}\text { Econo- } \\
\text { mía y } \\
\text { Desarro- } \\
\text { llo Sus- } \\
\text { tentable }\end{array}$ & $\begin{array}{l}\text { Historia } \\
\text { de } \\
\text { América } \\
\text { Colonial }\end{array}$ & $\begin{array}{l}\text { Geosiste } \\
\text { mas } \\
\text { Natura- } \\
\text { les II }\end{array}$ & $\begin{array}{l}\text { Historia } \\
\text { Medieval }\end{array}$ & $\begin{array}{l}\text { Curricu- } \\
\text { lum } \\
\text { Educa- } \\
\text { cional }\end{array}$ & $\begin{array}{l}\text { Formación } \\
\text { Integral } \\
\text { Oferta } \\
\text { Institu- } \\
\text { cional }\end{array}$ & $\begin{array}{l}\text { Inglés } \\
\text { Comuni- } \\
\text { cacional } \\
\text { I (FIP) }\end{array}$ \\
\hline $\begin{array}{l}\text { IV } \\
\text { Semes- } \\
\text { tre }\end{array}$ & $\begin{array}{l}\text { Riesgos y } \\
\text { desastres } \\
\text { naturales }\end{array}$ & $\begin{array}{l}\text { Historia } \\
\text { de Chile } \\
\text { Colonial }\end{array}$ & $\begin{array}{l}\text { Geografí } \\
\text { a Regio- } \\
\text { nal }\end{array}$ & $\begin{array}{l}\text { Historia } \\
\text { Moderna }\end{array}$ & $\begin{array}{l}\text { Didácti- } \\
\text { ca Gene- } \\
\text { ral }\end{array}$ & $\begin{array}{l}\text { Aproxi- } \\
\text { mación al }\end{array}$ & $\begin{array}{l}\text { Inglés } \\
\text { Comuni- }\end{array}$ \\
\hline
\end{tabular}




\begin{tabular}{|c|c|c|c|c|c|c|c|}
\hline & & & & & & $\begin{array}{l}\text { Sistema } \\
\text { Escolar }\end{array}$ & $\begin{array}{l}\text { cacional } \\
\text { II (FIP) }\end{array}$ \\
\hline $\begin{array}{l}\text { V } \\
\text { Semes- } \\
\text { tre }\end{array}$ & $\begin{array}{l}\text { Historia } \\
\text { de Chile } \\
\text { del siglo } \\
\text { XIX }\end{array}$ & $\begin{array}{l}\text { Historia } \\
\text { de } \\
\text { América } \\
\text { del siglo } \\
\text { XIX }\end{array}$ & $\begin{array}{l}\text { Geogra- } \\
\text { fía Re- } \\
\text { gional de } \\
\text { América }\end{array}$ & $\begin{array}{l}\text { Historia } \\
\text { Mundial } \\
\text { siglo XIX }\end{array}$ & $\begin{array}{l}\text { Evalua- } \\
\text { ción para } \\
\text { el Apren- } \\
\text { dizaje }\end{array}$ & $\begin{array}{l}\text { Ambientes } \\
\text { de Apren- } \\
\text { dizaje }\end{array}$ & $\begin{array}{l}\text { Inglés } \\
\text { Comuni- } \\
\text { cacional } \\
\text { III (FIP) }\end{array}$ \\
\hline $\begin{array}{l}\text { VI } \\
\text { Semes- } \\
\text { tre }\end{array}$ & $\begin{array}{l}\text { Historia } \\
\text { de Chile } \\
\text { Actual }\end{array}$ & $\begin{array}{l}\text { Historia } \\
\text { de Amé- } \\
\text { rica } \\
\text { Contem- } \\
\text { poránea }\end{array}$ & $\begin{array}{l}\text { Sistema } \\
\text { Natural } \\
\text { Chileno }\end{array}$ & $\begin{array}{l}\text { Historia } \\
\text { Mundial } \\
\text { Contempo } \\
\text { ránea }\end{array}$ & $\begin{array}{l}\text { Gestión } \\
\text { Educa- } \\
\text { cional y } \\
\text { Lideraz- } \\
\text { go Esco- } \\
\text { lar }\end{array}$ & $\begin{array}{l}\text { Trabajo } \\
\text { Colabora- } \\
\text { tivo en } \\
\text { Aula }\end{array}$ & $\begin{array}{l}\text { Inglés } \\
\text { Comuni- } \\
\text { cacional } \\
\text { IV (FIP) }\end{array}$ \\
\hline $\begin{array}{l}\text { VII } \\
\text { Semes- } \\
\text { tre }\end{array}$ & $\begin{array}{l}\text { Memoria } \\
\text { Histórica } \\
\text { y Ciuda- } \\
\text { danía }\end{array}$ & $\begin{array}{l}\text { Problemá- } \\
\text { ticas So- } \\
\text { ciales de } \\
\text { América y } \\
\text { Chile } \\
\text { Contem- } \\
\text { poráneo }\end{array}$ & $\begin{array}{l}\text { Sistema } \\
\text { Humano } \\
\text { Chileno }\end{array}$ & $\begin{array}{l}\text { Historia } \\
\text { Regional }\end{array}$ & $\begin{array}{l}\text { Investi- } \\
\text { gación } \\
\text { Educa- } \\
\text { cional }\end{array}$ & $\begin{array}{l}\text { Orienta- } \\
\text { ción Edu- } \\
\text { cacional y } \\
\text { Conviven- } \\
\text { cia Escolar }\end{array}$ & $\begin{array}{l}\text { Práctica } \\
\text { de Inves- } \\
\text { tigación } \\
\text { en Aula }\end{array}$ \\
\hline $\begin{array}{l}\text { VIII } \\
\text { Semes- } \\
\text { tre }\end{array}$ & $\begin{array}{l}\text { Didáctica } \\
\text { de la } \\
\text { Historia }\end{array}$ & $\begin{array}{l}\text { Metodo- } \\
\text { logía de la } \\
\text { Investiga- } \\
\text { ción Dis- } \\
\text { ciplinar }\end{array}$ & $\begin{array}{l}\text { Didáctica } \\
\text { de la } \\
\text { Geogra- } \\
\text { fía }\end{array}$ & $\begin{array}{l}\text { Ciudada- } \\
\text { nía y su } \\
\text { Enseñan- } \\
\text { za }\end{array}$ & $\begin{array}{l}\text { Proyecto } \\
\text { de Grado }\end{array}$ & $\begin{array}{l}\text { Inclusión } \\
\text { y Necesi- } \\
\text { dades } \\
\text { Educativas } \\
\text { Especiales }\end{array}$ & $\begin{array}{l}\text { Práctica } \\
\text { pedagó- } \\
\text { gica }\end{array}$ \\
\hline $\begin{array}{l}\text { IX } \\
\text { Semes } \\
\text { tre }\end{array}$ & $\begin{array}{l}\text { Activi- } \\
\text { dad de } \\
\text { Titula- } \\
\text { ción I }\end{array}$ & & & & & & $\begin{array}{l}\text { Práctica } \\
\text { Profesio- } \\
\text { nal }\end{array}$ \\
\hline
\end{tabular}

\section{Hito de monitoreo y evaluación}

El hito integrador o asignatura integradora es un mecanismo que forma parte de los procesos de monitoreo y evaluación de los perfiles de egreso y es un requerimiento de los procesos de acreditación para las carreras de pedagogía. Los hitos se recomiendan para dar cuenta del perfil (Mateo y Vlachopoulos, 2013; Alvarado et al., 2017). En los procesos de acreditación, la dimensión de "Propósitos e institucionalidad de la carrera o programa" 
(primera dimensión), en los descriptores $3 \mathrm{~h}$ y $4 \mathrm{e}$ de los criterios de "Perfil de egreso" y "Plan de estudios", exige como estándar contar con mecanismos sistematizados y documentados de monitoreo y evaluación que evidencien el alcance del perfil de egreso, así como mecanismos de evaluación de ciclo o procesos que permitan diseñar mejoras. Asimismo, la tercera dimensión del proceso de acreditación ("Resultados y capacidad de autorregulación"), en su criterio de "Efectividad y resultados de proceso formativo", exige mecanismos de evaluación para verificar el logro de perfiles, considerando la práctica. Estos antecedentes han llevado a que las carreras definan hitos, no obstante, es pertinente definir una estrategia para su diseño, evaluación y retroalimentación.

El hito es una instancia de evaluación integrada, una actividad curricular que debe incorporar las competencias del perfil de egreso, tanto genéricas como de especialidad, incluyendo las de carácter pedagógico, y debe ejecutarse en asignaturas del plan de estudios en los niveles inicial, intermedio y avanzado. Es pertinente un enfoque donde entendamos que las competencias se despliegan en el itinerario a lo largo de la carrera y van configurando procesos complejos de aprendizaje en todas las áreas del saber (conceptual, procedimental y actitudinal), hasta conformar una capacidad de despliegue y transferencia (Catalano et al., 2004).

Siguiendo lo anterior, se propone una estructura simple de diseño que permita hacer un ejercicio efectivo y que considere el tiempo de diseño como un factor clave de la gestión del currículum. En muchas ocasiones las propuestas de diseño curricular implican numerosas jornadas de dedicación, las cuales no siempre facilitan el trabajo colaborativo, la evidencia del desempeño ni la retroalimentación. Es necesario que estos ejercicios se compatibilicen con las otras funciones que se exigen a académicos/as y profesores/as.

El primer paso es definir las asignaturas donde se concentra el hito acorde a los tres momentos, como se observa en la Tabla 3, en las asignaturas señaladas con un marco rojo. En este caso la carrera, como en general sucede, determinó que el mejor escenario para los hitos son asignaturas donde se movilizan justamente todas las competencias y donde se requiere de desempeños para evidenciar el alcance del perfil. Así, la carrera incluyó la asignatura de práctica Aproximación al Sistema Escolar como hito 1. El hito 2 es la asignatura Laboratorio de la Didáctica de la Matemática y el hito 3 es la asignatura de Práctica Profesional. 


\section{Tabla 3}

Hitos evaluativos

\begin{tabular}{|c|c|c|c|c|c|c|c|}
\hline $\begin{array}{l}\text { I } \\
\text { Seme } \\
\text { stre }\end{array}$ & $\begin{array}{l}\text { Geome- } \\
\text { tría } \\
\text { Plana }\end{array}$ & $\begin{array}{l}\text { Álgebra } \\
\text { Elemental }\end{array}$ & $\begin{array}{l}\text { Taller de } \\
\text { Integra- } \\
\text { ción en } \\
\text { Resolu- } \\
\text { ción de } \\
\text { Problemas }\end{array}$ & $\begin{array}{l}\text { Psicología } \\
\text { Educacio- } \\
\text { nal }\end{array}$ & $\begin{array}{l}\text { Tecnolo- } \\
\text { gías para } \\
\text { el Apren- } \\
\text { dizaje }\end{array}$ & $\begin{array}{l}\text { Compe- } \\
\text { tencias } \\
\text { Comuni- } \\
\text { cativas FIP }\end{array}$ & $\begin{array}{l}\text { Inglés } \\
\text { Comu- } \\
\text { nicacio } \\
\text { nal I } \\
\text { (FIP) }\end{array}$ \\
\hline $\begin{array}{l}\text { II } \\
\text { Seme } \\
\text { stre }\end{array}$ & $\begin{array}{l}\text { Geome- } \\
\text { tría del } \\
\text { Espacio }\end{array}$ & $\begin{array}{l}\text { Álgebra y } \\
\text { Trigono- } \\
\text { metría }\end{array}$ & $\begin{array}{l}\text { Didáctica } \\
\text { Funda- } \\
\text { mental de } \\
\text { la Mate- } \\
\text { mática }\end{array}$ & $\begin{array}{l}\text { Sociología } \\
\text { de la Edu- } \\
\text { cación con } \\
\text { Enfoque } \\
\text { de Género }\end{array}$ & $\begin{array}{l}\text { Filosofía } \\
\text { Educacio- } \\
\text { nal }\end{array}$ & $\begin{array}{l}\text { Inglés } \\
\text { Comuni- } \\
\text { cacional II } \\
\text { (FIP) }\end{array}$ & \\
\hline $\begin{array}{l}\text { III } \\
\text { Seme } \\
\text { stre }\end{array}$ & $\begin{array}{l}\text { Geome- } \\
\text { tría } \\
\text { Analíti- } \\
\text { ca }\end{array}$ & $\begin{array}{l}\text { Cálculo } \\
\text { Diferen- } \\
\text { cial }\end{array}$ & $\begin{array}{l}\text { Aritméti- } \\
\text { ca }\end{array}$ & $\begin{array}{l}\text { Didáctica } \\
\text { de los } \\
\text { Sistemas } \\
\text { Numéricos }\end{array}$ & $\begin{array}{l}\text { Sociedad } \\
\text { Cultura y } \\
\text { Educación }\end{array}$ & $\begin{array}{l}\text { Inglés } \\
\text { Comuni- } \\
\text { cacional } \\
\text { III (FIP) }\end{array}$ & \\
\hline $\begin{array}{l}\text { IV } \\
\text { Seme } \\
\text { stre }\end{array}$ & $\begin{array}{l}\text { Didácti- } \\
\text { ca de la } \\
\text { Geome- } \\
\text { tría }\end{array}$ & $\begin{array}{l}\text { Cálculo } \\
\text { Integral }\end{array}$ & $\begin{array}{l}\text { Álgebra } \\
\text { Lineal }\end{array}$ & $\begin{array}{l}\text { Currícu- } \\
\text { lum Edu- } \\
\text { cacional }\end{array}$ & $\begin{array}{l}\text { Inglés } \\
\text { Comuni- } \\
\text { cacional } \\
\text { IV (FIP) }\end{array}$ & $\begin{array}{l}\text { Aproxima- } \\
\text { ción al } \\
\text { Sistema } \\
\text { Escolar }\end{array}$ & \\
\hline $\begin{array}{l}\text { V } \\
\text { Seme } \\
\text { stre }\end{array}$ & $\begin{array}{l}\text { Estadís- } \\
\text { tica y } \\
\text { Probabi- } \\
\text { lidades }\end{array}$ & $\begin{array}{l}\text { Cálculo en } \\
\text { Varias } \\
\text { Variables }\end{array}$ & $\begin{array}{l}\text { Razona- } \\
\text { miento en } \\
\text { Matemá- } \\
\text { tica }\end{array}$ & $\begin{array}{l}\text { Errores y } \\
\text { Obstáculos } \\
\text { del Apren- } \\
\text { dizaje en } \\
\text { Matemáti- } \\
\text { ca }\end{array}$ & $\begin{array}{l}\text { Didáctica } \\
\text { General }\end{array}$ & $\begin{array}{l}\text { Ambientes } \\
\text { de Apren- } \\
\text { dizaje }\end{array}$ & \\
\hline $\begin{array}{l}\text { VI } \\
\text { Seme } \\
\text { stre }\end{array}$ & $\begin{array}{l}\text { Inferen- } \\
\text { cia Es- } \\
\text { tadística }\end{array}$ & $\begin{array}{l}\text { Estructu- } \\
\text { ras Alge- } \\
\text { braicas }\end{array}$ & $\begin{array}{l}\text { Didáctica } \\
\text { del Álge- } \\
\text { bra y de } \\
\text { las Fun- } \\
\text { ciones }\end{array}$ & $\begin{array}{l}\text { Evaluación } \\
\text { para el } \\
\text { Aprendiza- } \\
\text { je }\end{array}$ & $\begin{array}{l}\text { Gestión } \\
\text { Educacio- } \\
\text { nal y Lide- } \\
\text { razgo } \\
\text { Escolar }\end{array}$ & $\begin{array}{l}\text { Trabajo } \\
\text { colaborati- } \\
\text { vo en Aula }\end{array}$ & \\
\hline $\begin{array}{l}\text { VII } \\
\text { Seme } \\
\text { stre }\end{array}$ & $\begin{array}{l}\text { Didácti- } \\
\text { ca de la } \\
\text { Estadís- } \\
\text { tica }\end{array}$ & $\begin{array}{l}\text { Ecuacio- } \\
\text { nes Dife- } \\
\text { renciales }\end{array}$ & $\begin{array}{l}\text { Historia y } \\
\text { Epistemo- } \\
\text { logía de la }\end{array}$ & $\begin{array}{l}\text { Tecnología } \\
\text { y Enseñan- } \\
\text { za de la }\end{array}$ & $\begin{array}{l}\text { Investiga- } \\
\text { ción Edu- } \\
\text { cacional }\end{array}$ & $\begin{array}{l}\text { Formación } \\
\text { Integral } \\
\text { Oferta }\end{array}$ & $\begin{array}{l}\text { Práctica } \\
\text { de } \\
\text { Inves- }\end{array}$ \\
\hline
\end{tabular}




\begin{tabular}{|c|c|c|c|c|c|c|c|}
\hline & & & $\begin{array}{l}\text { Matemá- } \\
\text { tica }\end{array}$ & $\begin{array}{l}\text { Matemáti- } \\
\text { ca }\end{array}$ & & $\begin{array}{l}\text { Institucio- } \\
\text { nal }\end{array}$ & $\begin{array}{l}\text { tigación } \\
\text { en Aula }\end{array}$ \\
\hline $\begin{array}{l}\text { VIII } \\
\text { Seme } \\
\text { stre }\end{array}$ & $\begin{array}{l}\text { Electivo } \\
\text { I }\end{array}$ & Electivo II & $\begin{array}{l}\text { Laborato- } \\
\text { rio de la } \\
\text { Didáctica } \\
\text { Matemá- } \\
\text { tica }\end{array}$ & $\begin{array}{l}\text { Proyecto } \\
\text { de Grado }\end{array}$ & $\begin{array}{l}\text { Orienta- } \\
\text { ción Edu- } \\
\text { cacional y } \\
\text { Conviven- } \\
\text { cia Escolar }\end{array}$ & $\begin{array}{l}\text { Inclusión y } \\
\text { Necesida- } \\
\text { des Educa- } \\
\text { tivas Espe- } \\
\text { ciales }\end{array}$ & $\begin{array}{l}\text { Práctica } \\
\text { Pedagó- } \\
\text { gica }\end{array}$ \\
\hline $\begin{array}{l}\text { IX } \\
\text { Seme } \\
\text { stre }\end{array}$ & $\begin{array}{l}\text { Forma- } \\
\text { ción } \\
\text { Integral } \\
\text { Oferta } \\
\text { Extra } \\
\text { Progra- } \\
\text { mática }\end{array}$ & & & & & $\begin{array}{l}\text { Actividad } \\
\text { Titulación } \\
\text { I }\end{array}$ & $\begin{array}{l}\text { Práctica } \\
\text { Profe- } \\
\text { sional }\end{array}$ \\
\hline $\begin{array}{l}\mathrm{X} \\
\text { Seme } \\
\text { stre }\end{array}$ & $\begin{array}{l}\text { Electivo } \\
\text { III }\end{array}$ & $\begin{array}{l}\text { Electivo } \\
\text { IV }\end{array}$ & $\begin{array}{l}\text { Electivo } \\
\mathrm{V}\end{array}$ & $\begin{array}{l}\text { Electivo } \\
\text { VI }\end{array}$ & & $\begin{array}{l}\text { Actividad } \\
\text { Titulación } \\
\text { II }\end{array}$ & \\
\hline
\end{tabular}

No podemos dejar de comentar el realce de la formación práctica que ha sido generado por las exigencias, criterios y marco conceptual de los procesos de acreditación. La formación práctica se ha consolidado como el escenario donde confluyen las formaciones teóricas y la imagen ideal del desempeño, con la realidad social externa y objetiva. Por ello, en general, las carreras han aumentado considerablemente las asignaturas y horas destinadas a evaluar bajo criterios de progresión, diversidad e integración curricular, donde las distintas líneas curriculares se orientan bajo el perfil de egreso (Zabalza, 2006). Las prácticas pasan asumir el rol fundamental de articulación de las áreas de formación y el desempeño de los estudiantes. Incluso se convierten en el escenario donde se evidencia el vínculo existente entre las carreras, los establecimientos educacionales y el contexto social.

Continuando con el hito de monitoreo y evaluación, el segundo paso es la definición de los desempeños a evaluar en cada hito. Nuestra propuesta apunta a un diseño de evaluación integrada, bajo una perspectiva constructivista, desde los resultados de aprendizaje más desafiantes en las asignaturas, teniendo como corte el hito pertinente. La metodología del levantamiento del desempeño contribuirá también a monitorear la coherencia y alineación del diseño curricular, al revisar cada una de las asignaturas desde 
una perspectiva de evaluación integradora, revisando incluso la tributación y alineamiento constructivo de las distintas áreas de formación, teniendo como base la taxonomía utilizada en el diseño. A continuación, un esquema que refleja este ejercicio.

\section{Figura 1}

Diseño de hito evaluativo

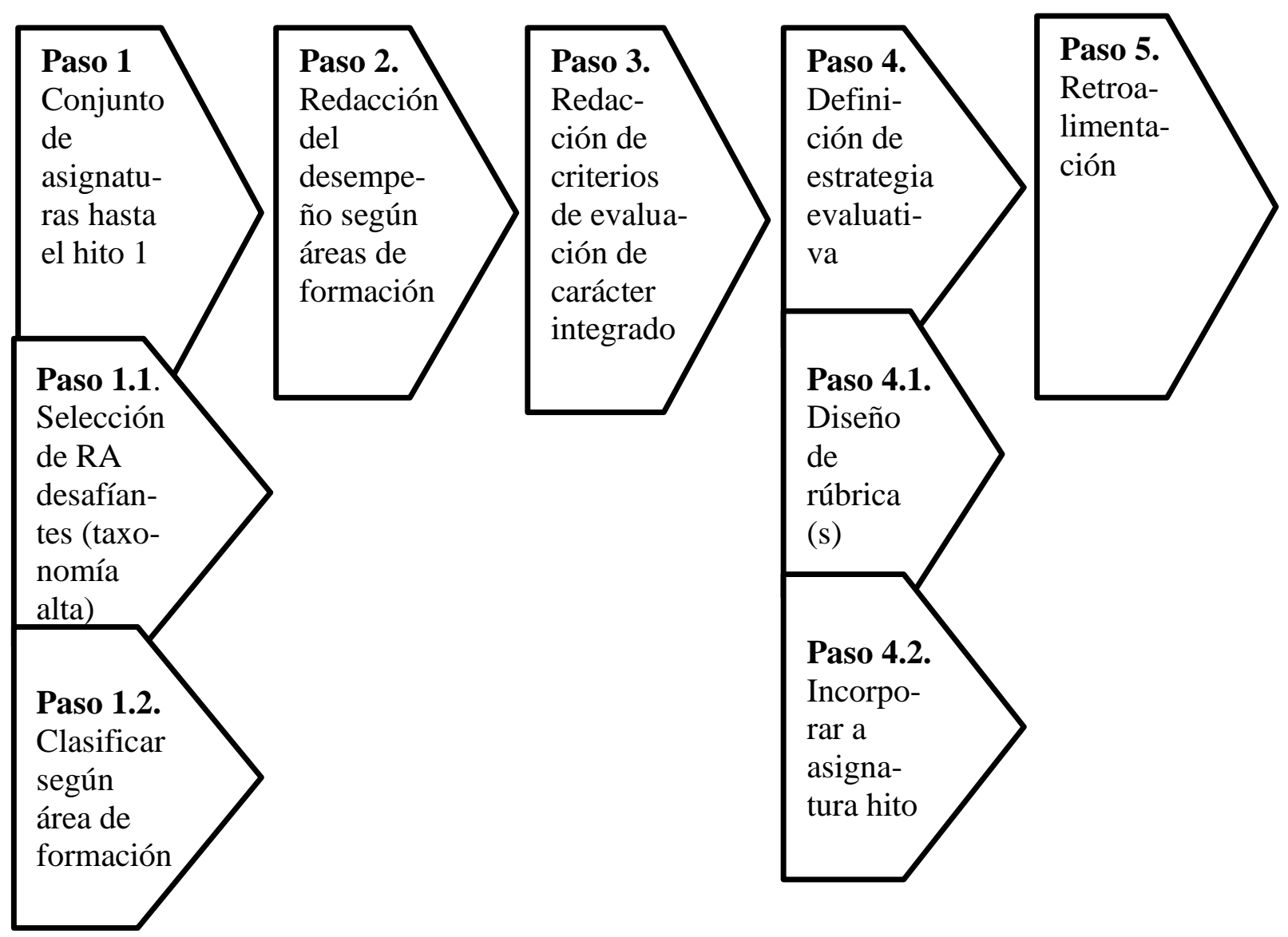

En el primer paso, se debe considerar el conjunto de las asignaturas hasta llegar al hito respectivo, donde específicamente se deben seleccionar los resultados de aprendizaje más desafiantes, que corresponden a taxonomías altas y que reflejan las áreas formativas (conceptual, procedimental y actitudinal), orientando así desde un principio el carácter integral del hito. La lectura de los resultados de aprendizaje con una mirada de revisión del perfil de egreso faculta a las escuelas para una revisión de sus matrices de tributación, como evidencia de diseño coherente, integradas y progresivas. Se recomienda el uso de 
las taxonomías cognitivas de Bloom, Anderson, Krathwohl y Marzano, la taxonomía psicomotora/procedimental de Simpson y la taxonomía actitudinal de Krathwohl, considerando tanto el verbo inicial como el de finalidad del resultado de aprendizaje.

Teniendo esta clasificación, se debe diseñar un desempeño por área formativa, esto es, un desempeño para el área de especialidad, el área de formación pedagógica y el área de formación integral. En el área de especialidad se podrá redactar más de un desempeño. La redacción de este desempeño tendrá la misma estructura que una competencia (verbo en $3^{\text {a }}$ persona singular + objeto + condición + finalidad).

Como tercer paso, teniendo los desempeños, se procede a determinar un conjunto de criterios de evaluación común a los desempeños redactados, los cuales van a orientar la estrategia evaluativa. Por consiguiente, es en los criterios de evaluación donde se estructura una evaluación integrada, tanto de las áreas de formación (disciplinar, pedagógica y formación integral) como de las áreas de conocimiento (conceptual, procedimental y actitudinal).

En el cuarto paso se define la estrategia de evaluación, la cual evidentemente debe ser coherente con la asignatura y el momento de evaluación (inicial, intermedio, avanzado). Este paso debe tener como evidencia el diseño de las rúbricas pertinentes. En general, se determinan instancias evaluativas complejas como el diseño de portafolios, estudios de caso, simulaciones, etc. Este hito debe tener un peso importante en el porcentaje de la evaluación de la asignatura, motivando a el/la estudiante a realizar el mayor esfuerzo posible. Conjuntamente, se debe considerar el contexto, lo que implica revisar la experiencia de los/as estudiantes en la estrategia definida, la capacidad de aplicación por parte de académicos/as y profesores/as, y los recursos físicos o de infraestructura requeridos. Es relevante que la forma de evaluar permita levantar un análisis del alcance del perfil de egreso, en términos de reportar el alcance de las competencias.

Para finalizar, tras la obtención de los resultados de la aplicación del hito y su estrategia evaluativa integradora, se debe convocar a un consejo de escuela, donde, bajo la lógica de círculo de aprendizaje, se analicen los resultados de los hitos y otros mecanismos de monitoreo y evaluación del perfil. Cabe recordar que el hito es un mecanismo complementario, que se suma a otros mecanismos como encuestas de satisfacción, 
informes de profesores/as tutores/as (en el caso de asignaturas prácticas), resultados en asignaturas (aprobación, reprobación, etc.), resultados de diagnósticos u otros mecanismos que las carreras determinen.

Es clave que los resultados se retroalimenten con estrategias para los ajustes curriculares, cambios metodológicos, planes de desarrollo extracurricular, fortalecimiento de capacitación de académicos/as y profesores/as, creación de líneas de investigación para formación docente, diseño de programas de formación continua, etc.

Así, el hito viene a conformar un sistema de evaluación integrado de los aprendizajes, en el cual se articula la evaluación del proceso formativo, incluyendo la planificación, implementación y ejecución, y se contribuye a la toma de decisiones para garantizar una gestión de calidad.

\section{Conclusión}

Hemos presentado hasta aquí una síntesis de las políticas públicas que presionan y exigen a las carreras de pedagogía un liderazgo en la gestión, que permita responder de manera sistemática a las exigencias del entorno. Se configuran nuevas estrategias de comunicación, transparencia y toma de decisiones que hacen que las carreras estén en un proceso de mejora y cambio permanente, lo que obliga a establecer mecanismos de gestión administrativa que no son simples de ejecutar en universidades públicas y estatales, donde los cambios curriculares, por ejemplo, necesitan ser aprobados y validados.

Las carreras de pedagogía necesitan adecuarse de manera rápida a la luz del estado del arte y los resultados de los mecanismos de monitoreo y evaluación implementados, donde los procesos de acreditación han jugado un papel importante en determinar las tareas necesarias. Dichos procesos vinieron a terminar un largo itinerario de exigencias, que comenzó con la implementación de renovaciones curriculares basadas en modelos por competencias que aún no terminaban de convencer y permear la forma de hacer las cosas, menos aún los criterios de desarrollo e implementación. Por ello, las instancias de 
acreditación constituyen un marco inicial de tareas, a las cuales las escuelas responden y que van cambiando lentamente las culturas institucionales.

En este contexto, se han diseñado dos mecanismos que forman parte de estrategias que vienen a superar debilidades de las carreras de pedagogía a nivel nacional, detectadas a través de los dictámenes de acreditación y los resultados de la Evaluación Nacional Diagnóstica II. Estas debilidades nos hablan, primero, de la dificultad de los/as estudiantes a la hora de la toma de decisiones didácticas en contextos de desempeño, así como de bajos resultados en temas de profesión docente. Asimismo, existen debilidades que hablan de una escasa integración de las distintas áreas formativas en los planes de estudio, incluso en programas de asignaturas que poseen una estructura por competencias, pero que no evalúan competencias.

El primer mecanismo diseñado para la superación de las debilidades enunciadas consiste en una propuesta que permite conformar un "itinerario de formación práctica", a través de la integración coherente de resultados de aprendizaje de carácter pedagógico y didáctico en asignaturas disciplinares, permitiendo con ello desarrollar la identidad docente y fortalecer la capacidad de toma de decisiones didácticas a la par del aprendizaje disciplinar. El segundo mecanismo propone una estructura metodológica para el diseño de un hito de evaluación integrada, que permita monitorear y evaluar el alcance del perfil de egreso. Este hito se configura a través del reconocimiento de la coherencia curricular de los resultados de aprendizaje y la definición de un desempeño y sus respectivos criterios de evaluación integrados. Esta evaluación debe ser analizada y debe orientar la toma de decisiones respecto a distintos aspectos, tales como las metodologías, los ajustes de diseño curricular, la conformación de líneas de investigación en formación inicial docente, actividades extracurriculares que fortalezcan debilidades o potencien fortalezas detectadas, e incluso el diseño de cursos o programas de formación continua.

Estos dos mecanismos nos plantean desafíos enormes para el liderazgo de la academia y los/as profesores/as formadores/as docentes, ya que implican acuerdo sobre principios de calidad, la responsabilidad por los resultados de los mecanismos y del proceso formativo, y el levantamiento de evidencia de la coherencia, integración y progresión del perfil de egreso y plan de estudio, haciendo transparente el análisis y la toma de decisiones. Ello también exige un trabajo colaborativo de académicos/as y profesores/as, 
disposición a la innovación y cambios en la didáctica y estrategia evaluativa, todo bajo una lógica de mejora continua, permanente y sistemática.

\section{Referencias}

Alvarado, M., Audibert, M., Cifuentes, C., Gómez, M., Mella, E. y Román, A. (2017). Evaluación del logro de perfiles de egreso: experiencias universitarias. Hitos evaluativos para evidenciar el logro de los perfiles. CINDA.

Amtmann, C. (2003). Nuevas demandas y nuevas respuestas de la universidad. ¿Una nueva crisis? En Centro Interuniversitario de Desarrollo-CINDA. Políticas públicas, demandas sociales y gestión del conocimiento (pp. 79-93). CINDA.

Beane, J. A. (1997). Curriculum integration: Designing the core of democratic education. Teachers College Press.

Bhola, Impara y Buckendahl (2005). Aligning Tests with States' Content Standards: Methods and Issues. Educational Measurement, 22 (3), 21-29. https://doi.org/10.1111/j.1745-3992.2003.tb00134.x

Biggs, J. (1996). Enhancing teaching through constructive alignment. Higher Education, 32(3), 347-364.

Bravo, N. H. (4 de diciembre de 2016). Competencias Proyecto Tuning-Europa, TuningAmérica Latina. Planeación curricular. https://planeacioncurricularpmi.wordpress.com/2016/12/04/competenciasproyecto-tuning-europa-tuning-america-latina/

Catalano, A., Avolio de Cols, S. y Sladogna, M. (2004). Diseño curricular basado en normas de competencia laboral. Banco Interamericano de Desarrollo.

Cisterna, C., Soto, V. y Rojas, C. (2016). Rediseño curricular en la Universidad de Concepción: la experiencia de las carreras de formación inicial docente. Calidad en la Educación, (44), 301-323. 
Comisión Nacional de Acreditación (2018). Barómetro del aseguramiento de la calidad de la educación superior $\quad$ (N 2$)$. https://investigacion.cnachile.cl/archivos/cna/documentos/c45_7ff_BarometroCNA-2.pdf

Comisión Nacional de Acreditación (2015). Criterios de evaluación para la acreditación de carreras profesionales, carreras profesionales con licenciatura y programas de licenciatura. https://www.cnachile.cl/Criterios\%20y\%20Procedimientos/DJ\%200094\%20Criterios.pdf

Comisión Nacional de Acreditación de Pregrado y Ministerio de Educación (2008). Manual de pares evaluadores: Guía para la evaluación externa con fines de acreditación. http://acreditaccion.cl/pdf/manual_pares_evaluadores.pdf

Centro de Perfeccionamiento, Experimentación e Investigaciones Pedagógicas. (2012). La política de formación inicial docente en CPEIP.

Centro de Perfeccionamiento, Experimentación e Investigaciones Pedagógicas (2012) Estándares Orientadores para Carreras de Pedagogía en Educación Media. https://www.cpeip.cl/wpcontent/uploads/2019/03/Est\%C3\%A1ndares_Media.p df

Centro de Perfeccionamiento, Experimentación e Investigaciones Pedagógicas (2019). Informe Resultados Evaluación Nacional Diagnóstica de la Formación Inicial Docente. Ministerio de Educación. https://www.cpeip.cl/wpcontent/uploads/2020/08/Informe-Nacional-END-2019_rect.pdf

Espinoza, O. (2014). Análisis crítico del discurso de las competencias en la formación inicial docente en Chile. Estudios Pedagógicos, 40 (2), 147-159. http://dx.doi.org/10.4067/S0718-07052014000300009

Ferreira, G. (2010). Hacia la integración curricular en la educación superior: reflexiones, necesidades y propuesta para la disciplina integradora. Revista Iberoamericana de Educación, 34 (2), 1- 10. 
González, J. y Wagenaar, R. (2003). Tunning-América Latina: Un Proyecto de las Universidades. Revista Iberoamericana de Educación (35), 151-164.

Hinde, E. T. (2005). Revisiting Curriculum Integration: A Fresh Look at an Old Idea. The Social Studies, 96(3), 105-111. https://doi.org/10.3200/TSSS.96.3.105-111

Hyland, T. (1994). Competence, Education and NVQS Dissenting Perspectives. Rewood Books.

Kri, F., Merchant, E., del Valle, R. Sánchez, T., Altieri, E., Ibarra, P., Vásquez M., Fernández F., Bravo C., Sánchez V., Salinas C. y Segovia, N. (2015). Manual para la implementación del Sistema de Créditos Académicos Transferibles SCTChile. Consejo de Rectores de las Universidades Chilenas.

Liu X. (2009). Beyond science Literacy: Science and the Public. International Journal of Environmental \& Science Education, 4, (3), 301-311.

López Quiroz, M. (2011). Criterios de coherencia y pertinencia para la evaluación inicial de planes y programas de pregrado: una propuesta teórico-metodológica. Revista de estudios y experiencia en educación, 10(19), 49-71.

Mateo, J. V. (2013). Reflexiones en torno al aprendizaje y a la evaluación en la universidad en el contexto de un nuevo paradigma para la educación superior. Educación XXI, 16(2), 183-208. https://doi.org/10.5944/educxx1.2.16.10338

Ministerio de Educación. (2012). Estándares Orientadores para Carreras de Pedagogía en Educación Media. https://www.cpeip.cl/wpcontent/uploads/2019/03/Est\%C3\%A1ndares_Media.pdf.

Ministerio de Educación. (2016). Marco Nacional de Cualificaciones para la Educación Superior. http://acreditaci.cl/wp-content/uploads/2017/06/MNC.pdf

Muller, J. (2009) Forms of knowledge and curriculum coherence. Journal of Education and Work, 22(3), 205-226. https://doi.org/10.1080/13639080902957905

Pedraja-Rejas L. M., Araneda-Guirriman, C. A., Rodríguez-Ponce, E. R. y RodríguezPonce J.J. (2012). Calidad en la Formación Inicial Docente: evidencia empírica 
en las universidades chilenas. Formación Universitaria, 5(4), 15-26. http://dx.doi.org/10.4067/S0718-50062012000400003

Penuel, W.; Fishman, B.; Gallagher, L.; Corbak, C. y López- Prado, B. (2009). Is Alignment Enough? investigating the Effects of State Policies and Professional Development on Science Curriculum Implementation. Science Education, Policy, 93 (4), 656-677, 2009. http://dx.doi.org/10.1002/sce.20321

Pey, R. y Chauriye, C. (2011). El Proceso de innovación curricular de las universidades del Consejo de Rectores. Consejo de Rectores de las Universidades Chilenas. http://sct-

chile.consejoderectores.cl/documentos_WEB/Innovacion_Curricular/2.Informe _INNOVACION_CURRICULAR.pdf

Restrepo, G., Ramos Reyes, J.A. y Sarmiento Lozano, J. (2000). Hacia unos fundamentos de la enseñanza y el aprendizaje de las ciencias sociales en la educación media. Universidad Nacional de Colombia.

Solar-Rodríguez, M. (2005). El currículum de competencias en la educación superior: desafíos y problemática. Pensamiento Educativo. Revista de Investigación Educacional Latinoamericana, 36(1), 172-191.

Tobón, S. (2006). Aspectos básicos de la formación basada en competencias. Talca: Proyecto MECESUP. [Archivo pdf] https://maristas.org.mx/gestion/web/doctos/aspectos_basicos_formacion_comp etencias.pdf

Tobón, S. (2009). Competencias en la Educación Superior. Políticas hacia la calidad. Ecoe Ediciones.

Unesco (1996). La educación encierra un tesoro. Informe a la Unesco de la Comisión Internacional sobre la educación para el siglo XXI, presidida por Jacques Delors. Unesco-Santillana.

Vecino Alegret, F. (1999). La Universidad a las puertas del nuevo siglo: una visión desde Cuba. Revista Bimestre Cubana, 85, 136-145. 
Verdejo, P, Forero, C, Burago, G. y Samoilovich, D. (2008). Propuestas y acciones universitarias para la transformación de la educación superior en América Latina. Informe final del proyecto $6 \times 4$ Uealc. Asociación Colombiana de Universidades-ASCUN.

Yániz, C., y Villardón, L. (2006). Planificar desde competencias para promover el aprendizaje. Cuadernos Monográficos del ICE, 12. Universidad de Deusto.

Zabalza, M. (2006). El practicum y la formación del profesorado: balance y propuesta para las nuevas titulaciones. En J.M. Escudero (Ed.), La mejora de la educación y la formación del profesorado. Políticas y prácticas (pp. 309-330). Octaedro. 